\title{
Mobility and Controllability of Bio-nanomachines
}

\author{
Yutaka Okaie \\ Graduate School of Frontier \\ Biosciences, Osaka University \\ Suita, Japan \\ yokaie@fbs.osaka- \\ u.ac.jp
}

\author{
Tadashi Nakano \\ Graduate School of Frontier \\ Biosciences, Osaka University \\ Suita, Japan \\ tadasi.nakano \\ @fbs.osaka-u.ac.jp
}

\author{
Takuya Obuchi \\ Graduate School of \\ Information Science and \\ Technology, Osaka University \\ Suita, Japan \\ obuchi.takuya@ist.osaka- \\ u.ac.jp
}

\author{
Takahiro Hara \\ Graduate School of \\ Information Science and \\ Technology, Osaka University \\ Suita, Japan \\ hara@ist.osaka-u.ac.jp
}

\author{
Shojiro Nishio \\ Graduate School of \\ Information Science and \\ Technology, Osaka University \\ Suita, Japan \\ nishio@ist.osaka-u.ac.jp
}

\begin{abstract}
Externally controllable molecular communication is expected to expand the capability of molecular communication by allowing conventional devices placed in the external environment to control the location and timing of molecular communication processes taking place in the molecular communication environment. In this paper, we consider externally controllable mobile bionanosensor networks where an external device is used to control the mobility of bio-nanomachines. We first describe a simple model to describe the mobility of bio-nanomachines. We then show how mutual information can be used to evaluate the controllability of mobile bio-nanomachines.
\end{abstract}

\section{Keywords}

Molecular communication, mobile bio-nanomachine, external control

\section{INTRODUCTION}

This paper applies the concept of externally controllable molecular communication [6] to design externally controllable mobile bionanosensor networks. A bionanosensor network considered in this paper consists of a group of mobile bio-nanomachines that communicate molecularly in the environment $[8,9,7]$ and an external device that controls the mobility of bio-nanomachines. Promising application areas of such networks include interactive drug delivery systems where human physicians use external control to direct drugcarrying bio-nanomachines to target locations in the body $[2,3]$.

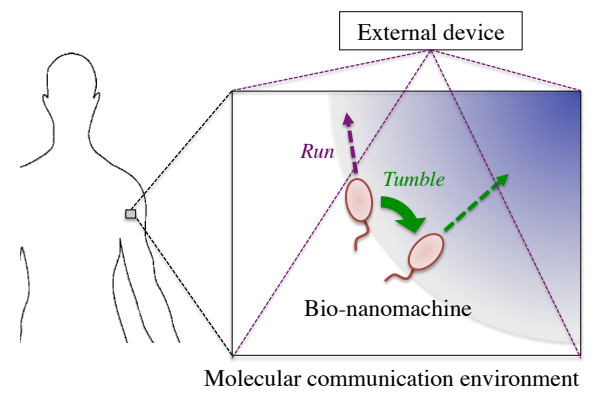

Figure 1: Interaction between an external device and a bio-nanomachine

Fig. 1 shows an overview of externally controllable mobile bionanosensor networks where the interaction between an external device and a bio-nanomachine is highlighted. An external device is a micro or larger scale conventional device (e.g., implantable and ingestible medical devices [4]) and it releases a type of attractant molecule to form a concentration gradient in the environment. A bio-nanomachine is a micro-scale device composed of biomaterials and exists in the molecular communication environment (e.g., in the human body). A bio-nanomachine has a chemotactic ability to detect the attractant concentration gradient and changes its moving direction based on the gradient. In the following, we first describe a simple stochastic model to describe the mobility of bio-nanomachines (Section 2). We then show how information theory may apply to evaluate the controllability of mobile bio-nanomachines (Section 3).

\section{MOBILITY MODEL}

We assume that an external device chooses a single point in a two-dimensional environment where a group of bionanomachines exist, releases a type of attractant molecule from the single point, and forms a concentration gradient in the environment. We also assume that each bio-nanomachine moves in a two-dimensional environment based on the mobility model of a chemotactic bacterium [1]. Briefly, each 
bio-nanomachine has two mobility modes: run and tumble modes. A bio-nanomachine in the run mode keeps its moving direction while a bio-nanomachine in the tumble mode changes its moving direction. A bio-nanomachine tends to move based on the run mode when moving up the attractant concentration gradient, otherwise it tends to move based on the tumble mode.

For a given bio-nanomachine in the environment, let $X$ be the direction of the incoming signal, representing the direction toward the point where the external device releases a type of attractant molecule (i.e., the direction of the maximum attractant concentration gradient at the location of the bio-nanomachine). For simplicity in the following, we assume that the direction $X$ follows $\mathcal{U}(-\pi, \pi)$. We further assume that the direction of the incoming signal $X$ is subject to noise in the environment; as a result, the bio-nanomachine receives the direction $\Theta$ that may follow Gaussian distribution $\mathcal{N}(x, \sigma)$ wrapped over a range $[x-\pi, x+\pi]$ where $\sigma$ represents noise effect.

Depending on the received signal $\theta$, a bio-nanomachine sets its mobility mode to either the run or tumble mode probabilistically based on the chemotactic index [5]. The bionanomachine then updates its moving direction $Y$; in the run mode, the bio-nanomachine retains its moving direction $Y$, and in the tumble mode it changes the moving direction $Y$ by $2 \pi / 81$ radian per second in either a clockwise or counter clockwise direction [1].

\section{PRELIMINARY RESULTS}

We use mutual information between $X$ and $Y$ to evaluate the controllability of mobile bio-nanomachines. Mutual information between $X$ and $Y$ dictates the distinct number of directions that the external device can choose to which bio-nanomachines are directed. In numerical experiments, we use the model described in the previous section, determine $Y$ after sufficiently long time elapses for a given $X$, and compute mutual information between $X$ and $Y$.

Fig. 2 shows the distribution of the moving direction $Y$ of a bio-nanomachine when the direction of the incoming signal $X$ is 0 with noise effect $\sigma$ varied in $\{0.1,0.5,1.0\}$. As shown in the figure, more bio-nanomachines are directed toward the direction $X$ as $\sigma$ decreases. Fig. 3 shows the impact of noise on mutual information between $X$ and $Y$. When $\sigma=0.1$, the mutual information is 1.79, meaning that $2^{1.79} \simeq 3.46$ distinct number of directions can be correctly communicated with the bio-nanomachine. As $\sigma$ increases, the mutual information decreases to around 0.09, showing how controllability decreases as the noise effect increases.

how information theory may apply to analyze the performance of bacterium-based bio-nanomachines.

\section{ACKNOWLEDGMENTS}

This work was supported by the Japan Society for the Promotion of Science (JSPS) through the Grants-in-Aid for Scientific Research (No. 25240011).

\section{REFERENCES}

[1] H. C. Berg and D. A. Brown. Chemotaxis in escherichia coli analysed by three-dimensional

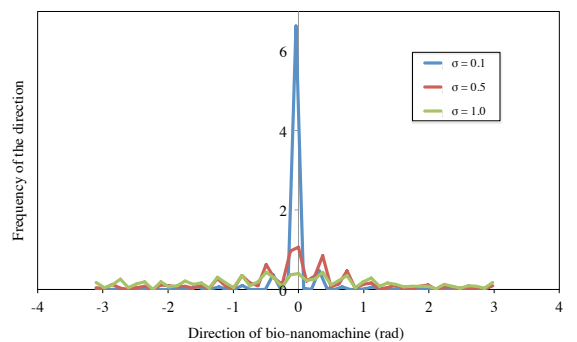

Figure 2: Impact of noise on moving directions

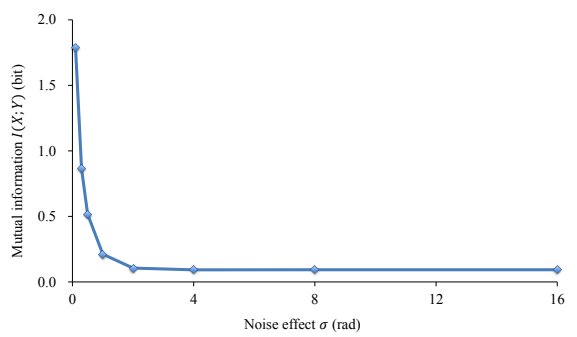

Figure 3: Impact of noise on mutual information

tracking. Nature, 239: 500-504, 1972.

[2] Y. Chen, P. Kosmas, P. S. Anwar, and L. Huang. A touchable molecular communication model of targeted contrast agent delivery. IEEE Internal Conference on Communications (ICC), 1091-1096, 2015.

[3] Y. Chen, P. Kosmas, P. S. Anwar, and L. Huang. A touch-communication framework for drug delivery based on a transient microbot system. IEEE Transactions on Nanobioscience, 14(4):397-408, 2015.

[4] A. Kiourti, K. A. Psathas, and K. S. Nikita. Implantable and ingestible medical devices with wireless telemetry functionalities: A review of current status and challenges. Bioelectromagnetics, 35(1):1-15, 2014.

[5] C. P. McCann, P. W. Kriebel, C. A. Parent, and W. Losert. Cell speed, persistence and information transmission during signal relay and collective migration. Journal of Cell Science, 123:1724-1731, 2010.

[6] T. Nakano, S. Kobayashi, T. Suda, Y. Okaie, Y. Hiraoka, and T. Haraguchi. Externally controllable molecular communication. IEEE Journal of Selected Areas in Communication (JSAC), 32(12):2417-2431, 2014.

[7] T. Obuchi, Y. Okaie, T. Nakano, T. Hara, and S. Nishio. Inbody mobile bionanosensor networks through non-diffusion-based molecular communication. IEEE International Conference on Communication (ICC), 1078-1084, 2015.

[8] Y. Okaie, T. Nakano, T. Hara, T. Obuchi, K. Hosoda, Y. Hiraoka, and S. Nishio. Cooperative target tracking by a bacterium-based mobile sensor network. IEEE Transactions on Nanobioscience, 13(3):267-277, 2014.

[9] G. Wei, P. Bogdan, and R. Marculescu. Bumpy rides: Modeling the dynamics of chemotactic interacting bacteria. IEEE Journal of Selected Areas in Communication (JSAC), 31(12):879-890, 2013. 